\title{
Direct observation and theoretical interpretation of strongly enhanced lateral diffusion of photogenerated carriers in InGaN/GaN quantum well structures
}

\author{
S. J. Xu, ${ }^{\text {a) }}$ Y. J. Wang, and Q. Li \\ Department of Physics and HKU-CAS Joint Laboratory on New Materials, The University of Hong Kong, \\ Pokfulam Road, Hong Kong, People's Republic of China
}

X. H. Zhang, W. Liu, and S. J. Chua

Institute of Materials Research and Engineering, 3 Research Link, Singapore 117602, Singapore

(Received 27 September 2004; accepted 7 December 2004; published online 8 February 2005)

\begin{abstract}
Strongly enhanced lateral diffusion of photogenerated carriers was directly observed in the luminescent image of the InGaN/GaN quantum wells. Such an effect was quantitatively modeled using diffusion equation and the ambipolar diffusion coefficient derived by K. H. Gulden and his co-workers [Phys. Rev. Lett. 66, 373 (1991)]. Our simulation shows that the vertical piezoelectric field existing in strained InGaN/GaN quantum wells is the original "driving force" for the enhancement of lateral diffusion. Influence of the density of photogenerated carriers and their average mobility on the enhancement was discussed. (C) 2005 American Institute of Physics.
\end{abstract}

[DOI: $10.1063 / 1.1862774$ ]

Strained InGaN/GaN quantum wells (QWs) play a critical role in newly developed GaN based short-wavelength light-emitting devices. ${ }^{1}$ The current status in the study of $\mathrm{GaN}$ area is that the empirical development in light-emitting devices is advancing rapidly while the fundamental understanding on many issues of materials is struggling to catch up and even still poor. ${ }^{2}$ One such issue poorly understood is the diffusion mechanism of photogenerated carriers in InGaN/GaN QW structures. Recently, Huang and his co-workers ${ }^{3}$ reported an investigation on the lateral ambipolar diffusion in InGaN/GaN multiple QWs using optical transient transmission measurements. They observed a large ambipolar diffusion coefficient in the structures and attributed this enhancement to the existence of strong piezoelectric field in the structures. They also found that the lateral ambipolar diffusion coefficient increases with increasing the InGaN well width. These behaviors observed in the InGaN/GaN QWs are very interesting and need to be quantitatively understood. In this letter we give a direct luminescent image evidence of the enhanced diffusion of photogenerated carriers in InGaN/GaN QWs and attempt to quantitatively simulate the phenomenon using diffusion equation and an analytical expression of the ambipolar diffusion coefficient derived by Gulden et al. ${ }^{4}$ The simulation results clearly demonstrate the original and central role of the piezoelectric field in the enhanced lateral diffusion of photogenerated carriers in the structures. The results also reveal that the density of photogenerated carriers and their average mobility have important influence on the enhanced lateral diffusion.

The InGaN/GaN QWs samples employed in the study were grown on sapphire with metalorganic vapor phase epitaxy. The typical growth sequence of the samples is $30 \mathrm{~nm}$ $\mathrm{GaN}$ nucleation layer grown at $500{ }^{\circ} \mathrm{C}, 1500 \mathrm{~nm} \mathrm{GaN}$ buffer at $1050{ }^{\circ} \mathrm{C}$, four periods of InGaN/GaN QWs at $740{ }^{\circ} \mathrm{C}$, and $20 \mathrm{~nm} \mathrm{GaN}$ cap layer at $740^{\circ} \mathrm{C}$. The experimental data

\footnotetext{
a) Author to whom correspondence should be addressed; electronic mail: sjxu@hkucc.hku.hk
}

of two representative samples 1622 and 1323 was reported in the work. Sample 1622 has 2-nm-thick wells and 12-nm-thick barriers while sample 1323 has 3.4-nm-thick wells and 3-nm-thick barriers. The photoluminescence spectroscopy system used in the study has been described previously. $^{5}$

Figure 1 shows digital images of light emission from samples 1622 and 1323 at $5 \mathrm{~K}$. We also took the digital luminescent images of the samples at room temperature. Very similar images were obtained (not shown here). The central white circular regions in the two images in Fig. 1 are the laser spots on the surface of samples while the turquoise areas surrounding the central excitation spots and the green or blue edge loops are the emission areas. In the laser excitation spots, the intensities of the luminescence signals are basically constants along their radial directions due to the uniform generation of the photogenerated carriers in these regions. It is clear that the light emission areas are much larger than the excitation spots. This unambiguously indicates that the strong lateral diffusion of photogenerated carriers takes place. It should be noted that the enlarged luminescent area strongly indicates the nature of ambipolar diffusion. In other words, both electrons and holes simultaneously diffuse due to the Coulomb attraction between both particles with opposite electrical charge. As pointed out by Yairi and Miller, ${ }^{6}$ a lateral electric field created by the verti-
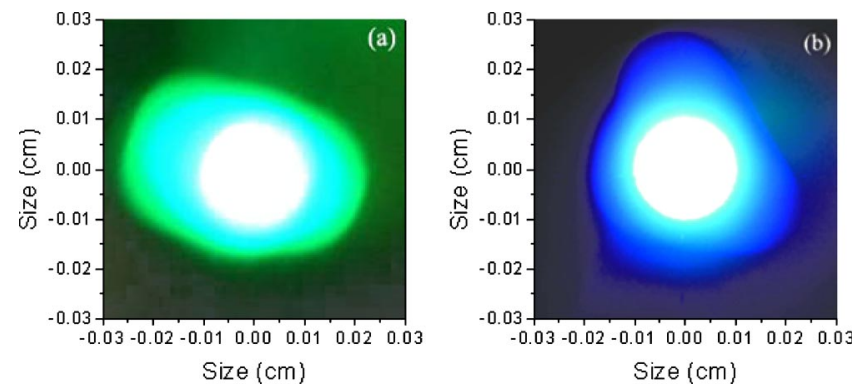

FIG. 1. (Color online). The luminescent images of two InGaN/GaN QW samples at $5 \mathrm{~K}$ under excitation of $325 \mathrm{~nm}$ UV laser; (a) is for sample 1622 and (b) for sample 1323. 


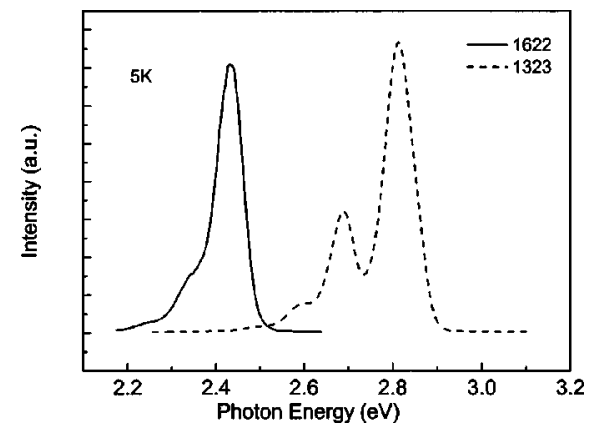

FIG. 2. The $5 \mathrm{~K}$ PL spectra of two InGaN/GaN QW samples.

cal separation of the photogenerated carriers due to the vertical built-in or externally applied electric field in semiconductor $p-i-n$ or $n-i-p-i$ structures results in the giant lateral diffusion. It is obvious that the strained $\mathrm{InGaN} / \mathrm{GaN} \mathrm{QW}$ structures possess the necessary conditions, i.e., the built-in strong piezoelectric field ${ }^{7,8}$ and efficient screening effect of photogenerated carriers on the piezoelectric field, ${ }^{9}$ for appearance of the giant lateral ambipolar diffusion. As shown in Fig. 1, the strong enhancement of lateral diffusion of photogenerated carriers was indeed observed in InGaN/GaN QWs. The corresponding photoluminescence (PL) spectra of two samples were depicted in Fig. 2.

When InGaN/GaN QWs are optically excited with a beam of $\mathrm{He}-\mathrm{Cd} 325 \mathrm{~nm}$ laser, the incident photons are absorbed in the QW region and excess electrons and holes are created. These photogenerated carriers will be vertically separated by the vertical piezoelectric field $E_{P}$ induced by strain. The separated carriers will produce a net field $E_{S}$ to screen the piezoelectric field. In the region where no incident light illuminated, the difference between the electron and hole quasi-Fermi levels, $\phi_{n p}$ is determined by the vertical piezoelectric field $E_{P}$. On the other hand, in the light excitation region where the incident light is absorbed, the vertically separated carriers screen the piezoelectric field $E_{P}$. As a result, $\phi_{n p}$ changes significantly because it is an approximately linear function of the separated photogenerated carrier density. ${ }^{6}$ Moreover, it has a lateral dependence due to the lateral intensity variation of the incident light beam. The resulting lateral gradients of both the electron and hole quasiFermi level, $\phi_{n}$ and $\phi_{p}$, produce electric fields in the top and bottom layers. These fields push both electrons and holes laterally away and make enhanced diffusion possible. In the following equations, the subscripts of $n$ and $p$ refer to electron and hole, respectively. The symbols of $j, e, n, p, D_{\text {am }}$, $\mu_{n}, \mu_{p}, L_{D}$, and $L_{Q}$ represent current density, the electronic charge, electron density, hole density, ambipolar diffusion coefficient, electron mobility, hole mobility, thickness of the electrically charged layer due to the existence of the strong piezoelectric field, and total thickness of the quantum wells including the barrier layers, respectively. The ambipolar diffusion coefficient $D_{\mathrm{am}}$ is defined by ${ }^{4}$

$$
j_{n}(\mathbf{r})=e D_{\mathrm{am}} \nabla n(\mathbf{r})=-j_{p}(\mathbf{r})=e D_{\mathrm{am}} \nabla p(\mathbf{r}) .
$$

Actually, the above equation also reflects such a fact that no net electrical current generates in the structures. As mentioned earlier, an analytical expression of the ambipolar diffusion coefficient $D_{\text {am }}$ was derived as follows: ${ }^{4}$ fusion coefficient $D_{\text {am }}$ was derived as follows: ${ }^{4} \quad(\rho, \theta)$. Here $\rho$ and $\theta$ represent in-plane radial distance and
Downloaded $20 \mathrm{Apr} 2007$ to 147.8.143.135. Redistribution subject to AlP license or copyright, see http://apl.aip.org/apl/copyright.jsp

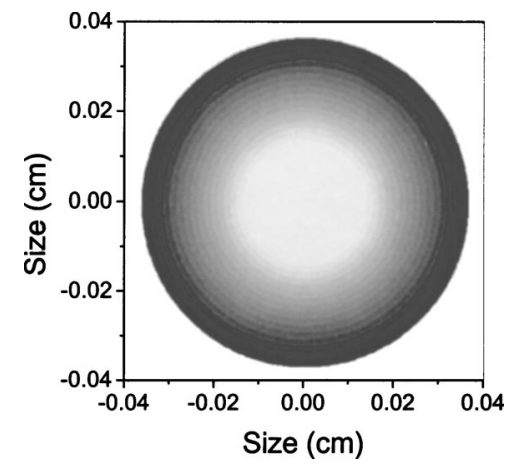

FIG. 3. Calculated in-plane diffusion region of photogenerated carriers in comparison with the excitation spot (white circular region).

$$
D_{\mathrm{am}}=\frac{\sigma_{n} \sigma_{p}}{\sigma_{n}+\sigma_{p}} \frac{\partial \phi_{n p}}{\partial n} \frac{1}{e^{2}},
$$

where $\sigma_{n}=n \mu_{n} e$ is electron conductivity and $\sigma_{p}=p \mu_{p} e$ hole conductivity. From expression (2), it can be seen that the ambipolar diffusion coefficient depends directly on how fast the energy difference is between the electron and hole quasiFermi levels with the density of separated photogenerated carriers. Once the quantity $\partial \phi_{n p} / \partial n$ is known the ambipolar diffusion coefficient $D_{\text {am }}$ can be obtained. The energy difference between the quasi-Fermi levels of electrons and holes has an approximated linear relationship with the screening electric field of photogenerated carriers. ${ }^{10}$ That is, $\phi_{n p}$ $\approx E_{S} L_{Q} e$.

For the strained InGaN/GaN QW structures optically illuminated, the net vertical electric field $E$ in the QW regions is given by $E_{P}-E_{S}$ and can be calculated from numerically solving Poisson's equation. ${ }^{9}$ Here we adopt an analytical expression of $E_{S}$ derived by Poole et al. ${ }^{10}$ to calculate $\phi_{n p}$ and then $\partial \phi_{n p} / \partial n$. Their expressions are given as follows:

$$
\begin{aligned}
& E_{s}=\frac{n}{2 \varepsilon_{r} \varepsilon_{0}} L_{D} e, \\
& \phi_{n p} \approx \frac{n}{2 \varepsilon_{r} \varepsilon_{0}} L_{D} L_{Q} e^{2}
\end{aligned}
$$

and

$$
\frac{\partial \phi_{n p}}{\partial n}=\frac{L_{D} L_{Q} e^{2}}{2 \varepsilon_{r} \varepsilon_{0}},
$$

where $\varepsilon_{r}$ and $\varepsilon_{0}$ are the relative dielectric constant of the InGaN and the permittivity of vacuum, respectively.

The diffusion equation describing the steady-state distribution of photogenerated carriers in InGaN/GaN QWs can be written as

$$
\frac{d n(\mathbf{r})}{d t}=-D_{a m} \nabla n(\mathbf{r})+\frac{n(\mathbf{r})}{\tau}=0,
$$

where $\tau$ is the lifetime of photogenerated carriers. Because the lateral distribution of the photogenerated carriers is only concerned in the present work Eq. (6) can be simplified. Furthermore, in the derivation of the ambipolar diffusion coefficient, $^{4,6}$ it was assumed that the photogenerated electrons and holes have already separated in the vertical direction across the intrinsic region of the $p-i-n$ structures. Therefore, $n$ and $p$ are functions of just the lateral dimensions 
TABLE I. Material parameters used in calculations.

Band-gap energy of GaN $E_{g}(\mathrm{eV})$

Dielectric constant of $\mathrm{GaN} \varepsilon$ (in $\varepsilon_{0}$ )

Mobility of electrons in $\mathrm{GaN} \mu_{n}\left(\mathrm{~cm}^{2} \mathrm{~V}^{-1} \mathrm{~s}^{-1}\right)$

Mobility of holes in GaN $\mu_{p}\left(\mathrm{~cm}^{2} \mathrm{~V}^{-1} \mathrm{~s}^{-1}\right)$

Lifetime of carriers in InGaN/GaN QWs $\tau$ (ns)

Thickness of electrically charged layer $L_{D}(\mathrm{~nm})$

Thickness of quantum wells region $L_{Q}(\mathrm{~nm})$

Reference 11 .

${ }^{\mathrm{b}}$ Reference 12 .

${ }^{\mathrm{c}}$ Reference 13.

${ }^{\mathrm{d}}$ Reference 14 .

polar angle, respectively. Equation (6) can thus be reduced to a second order ordinary differential equation as follows:

$$
n^{\prime \prime}(\rho)+\frac{1}{\rho} n^{\prime}(\rho)-\frac{n(\rho)}{D_{\mathrm{am}} \tau}=0 .
$$

In the numerical calculation of Eq. (7), the finite difference method was employed. The boundary conditions used were

$$
\begin{gathered}
n(\rho)=1.0 \times 10^{17} \mathrm{~cm}^{-3} \text { at } 0 \leqslant \rho \leqslant 0.01 \mathrm{~cm} ; \\
n(\rho)=0 \text { at } \rho=\infty .
\end{gathered}
$$

Figure 3 depicts the calculated in-plane radial distribution of photogenerated carriers in InGaN/GaN QWs structure. The parameters adopted in the calculation were listed in Table I. Clearly, the distribution area of photogenerated carriers is much larger than that of the excitation spot (just central white region). Here we defined the diffusion radius as the distance from the center to the edge where the density of carrier decreases to zero after a steady state builds up. Obviously, the calculated results are in good agreement with the experimental data shown in Fig. 1. The influence of photogenerated carrier density and average mobility [defined as $\left.\mu_{n} \mu_{p} /\left(\mu_{n}+\mu_{p}\right)\right]$ on the enhanced diffusion was also examined. Figure 4 shows the calculated diffusion radius of photogenerated carriers in InGaN/GaN QWs versus the average mobility of carriers. It can be seen that the diffusion radius superlinearly increases with increasing the average mobility. As expected, the larger the photogenerated carrier density the longer the in-plane diffusion radius. Substituting Eq. (5) into Eq. (2) yields $D_{\mathrm{am}} \propto\left(n L_{Q}\right)$, which can theoretically interpret behaviors observed by Huang and his co-workers. ${ }^{3}$ We also note that there is a possibility that the photogenerated carrier density in Huang's case may greatly differ from our case. In Huang's experiment, the frequency-doubled Ti:sapphire pulse laser was used to excite the samples and this could lead to much higher carrier density.

It is worth mentioning that in the calculations the inplane diffusion of photogenerated carriers is assumed to be isotropic. However, the in-plane diffusion may be anisotropic, as shown in Fig. 1. If the mobility of carriers in InGaN/GaN QWs with hexagonal crystalline structure is an anisotropic quantity, the diffusion radius of photogenerated carriers is certainly direction dependent. Another point we want to point out is that the strongly enhanced lateral diffusion of carriers may dramatically affect the performance of InGaN/GaN light-emitting devices with a small mesa structure, such as, it may lead to a bottleneck effect on the devices' output power. The effect of enhanced lateral diffusion

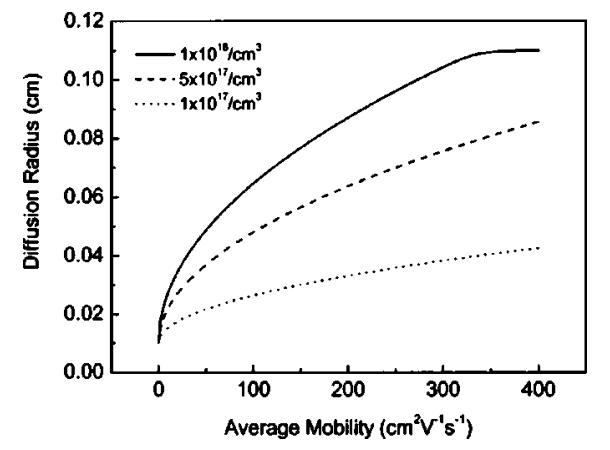

FIG. 4. Calculated diffusion radius of photogenerated carriers as a function of the average mobility of electrons and holes with different photogenerated carrier densities.

of photogenerated carriers in quantum structure itself is very interesting issue. The finding and confirmation of the effect in InGaN/GaN QW structures may make them have important potential applications. Moreover, studying this effect also offers a good opportunity to enhance understanding of carrier dynamics in InGaN/GaN QW structures.

In conclusion, strongly enhanced lateral diffusion of photogenerated carriers in InGaN/GaN QWs was certainly confirmed from the luminescent images. Theoretical simulation to the enhanced diffusion effect was presented. The calculated results quantitatively agree with the experimental data. It is shown that the lateral gradient of photogenerated carriers vertically separated by the piezoelectric field causes a lateral electrical field and then results in an enhancement of the lateral diffusion coefficient. The strongly enhanced lateral diffusion effect in InGaN/GaN QWs may have potential applications. At the same time, it might significantly affect the optical and even electrical characteristics of InGaN/GaN QWs light-emitting devices.

The work was supported by HK RGC CERG Grant No. HKU 7036/03P.

${ }^{1}$ S. Nakamura, S. Pearton, and G. Fasol, The Blue Laser Diode, 2nd ed. (Springer, Berlin, 2000).

${ }^{2}$ N. M. Johnson, A. V. Nurmikko, and S. P. DenBaars, Phys. Today 53, 31 (2000).

${ }^{3}$ Y.-C. Huang, J.-C. Liang, C.-K. Sun, A. Abare, and S. P. DenBaars, Appl. Phys. Lett. 78, 928 (2001).

${ }^{4}$ K. H. Gulden, H. Lin, P. Kiesel, P. Riel, G. H. Döhler, and K. J. Ebeling, Phys. Rev. Lett. 66, 373 (1991).

${ }^{5}$ S. J. Xu, W. Liu, and M. F. Li, Appl. Phys. Lett. 77, 3376 (2000).

${ }^{6}$ M. B. Yairi and D. A. B. Miller, J. Appl. Phys. 91, 4374 (2002).

${ }^{7}$ F. Bernardini, V. Fiorentini, and D. Vanderbilt, Phys. Rev. B 56, R10024 (1997).

${ }^{8}$ T. Takeuchi, C. Wetzel, S. Yamaguchi, H. Sakai, H. Amano, I. Akasaki, Y. Kaneko, S. Nakagawa, Y. Yamaoka, and N. Yamada, Appl. Phys. Lett. 73, 1691 (1998).

${ }^{9}$ Q. Li, S. J. Xu, M. H. Xie, S. Y. Tong, X. H. Zhang, W. Liu, and S. J. Chua, Jpn. J. Appl. Phys., Part 2 41, L1093 (2002).

${ }^{10}$ P. J. Poole, C. C. Phillips, M. Henini, and O. H. Hughes, Semicond. Sci. Technol. 8, 1750 (1993).

${ }^{11}$ I. Vurgaftman, J. R. Meyer, and L. R. Ram-Mohan, J. Appl. Phys. 89, 5815 (2001).

${ }^{12}$ V. Bougrov, M. E. Levinshtein, S. L. Rumyantsev, and A. Zubrilov, inProperties of Advanced Semiconductor Materials GaN, AlN, InN, BN, SiC, SiGe, edited by M. E. Levinshtein, S. L. Rumyantsev, and M. S. Shur (Wiley, New York, 2001), p. 1.

${ }^{13}$ T. P. Chow and M. Ghezzo, SiC power devices, in III-Nitride, SiC, and Diamond Materials for Electronic Devices, edited by D. K. Gaskill, C. D. Brandt, and R. J. Nemanich, Material Research Society Symposium Proceedings, Pittsburgh, PA, 1996, Vol. 423, p. 69.

${ }^{14}$ X. H. Zhang, S. J. Chua, W. Liu, and P. Li (unpublished). 